\title{
VALOR DA GAMAENCEFALOMETRIA NO DIAGNÓSTICO DAS EPILEPSIAS
}

\author{
Luís Marques de ASSIS * \\ JoÃo TeIXeIra PINTo **
}

A gamaencefalometria é um método relativamente novo na semiologia nervosa; baseia-se na maior absorção, por parte de tecido nervoso anormal, de isótopos que emitem radiação gama. Esse método tem sido usado no diagnóstico de tumores cerebrais, fornecendo dados quanto à localização e, às vêzes, quanto à natureza do processo patológico.

Raras têm sido as referências ao emprêgo da gamaencefalometria (GEM) nas epilepsias. Planiol e Fischgold ${ }^{2}$ utilizaram a GEM em 126 casos de epilepsia tardia no adulto, separando-os em três grupos: tumorais (diagnóstico confirmado por ato cirúrgico ou por exames radiológicos contrastados), não tumorais e de causa não determinada. Dos 48 casos tumorais, o EEG foi anormal em 43 e a GEM em 44; dos 55 não tumorais, o EEG foi anormal em 42 e a GEM em apenas 3 ; dos 23 de causa indeterminada, o EEG foi anormal em 16, não tendo a GEM mostrado qualquer anormalidade em nenhum dêles. Com base nesses dados os autores concluiram que a exploração cerebral pelos radioisótopos dá, nos casos de epilepsia condicionada pela existência de tumor intracraniano, $80 \%$ de probabilidade de acêrto quanto ao diagnóstico etiológico; por outro lado, nos casos de epilepsia de longa duração, a negatividade dêsse exame constitui uma segurança suplementar quanto à não existência de tumor cerebral.

\section{MATERIAL E METODO}

Foram estudados 42 pacientes epilépticos, com mais de 10 anos de idade, sem sinais neurológicos focais e sem hipertensão intracraniana.

Em 36 dos 42 casos estudados havia referências anamnésticas a episódios convulsivos; dêsse total, em 8 casos as convulsões eram, clinicamente, do tipo centrencefálico; em 23 representavam a generalização de crise focal; em 5 as convulsôes ocorriam apenas durante o sono. Em 6 casos não havia, na anamnese, referência a fenômenos convulsivos. O exame do líqüido cefalorraquidiano, feito em 40 pacientes, foi normal em todos. O pneumencefalograma foi normal em um dos dois casos em que foi feito. O eletrencefalograma, feito em todos os casos, foi anormal em 36, sendo a anormalidade de caráter difuso em 6 e focal nos demais.

Os pacientes ingeriam 30 gôtas de Lugol por via oral, diàriamente, nos dois dias que precediam o exame gamaencefalométrico. Foi empregada a RHISA (albu-

Trabalho da Clínica Neurológica da Fac. Med. da Univ. de São Paulo (Prof. Adherbal Tolosa). * Médico contrałado; * Chefe da Seccão de Radioisótopos. 
mina humana marcada com iôdo radioativo) nas doses de 300 a 350 microcuries, por via intravenosa. As medições foram feitas 24 e 48 horas após a injeção do isótopo, sendo lançadas em gráfico. Foram obtidos dois tipos de alterações; $n \cdot \jmath$ primeiro, tipo edema, a absorção é alta nas primeiras 24 horas, caindo de maneira sensivel nas 24 horas seguintes; no segundo, tipo lesão, a absorção permanece alta nas 48 horas após a injeção da RHISA.

A aparelhagem utilizada foi fornecida pela Berkeley: um cintilador (cabeça de $1,5 \times 1,5$ polegadas) e um medidor de impulsos (ratemeter).

\section{RESULTADOS}

A GEM foi anormal em 25 dos 42 casos estudados. Os quadros 1,2 e 3 corre. lacionam as anormalidades encontradas com o tempo de doença, com os resultados dos exames eletrencefalográficos e com o quadro clínico.

\begin{tabular}{c|c|c}
\hline Tempo de doença (anos) & Número de casos & $\begin{array}{c}\text { Gamaencefalometria } \\
\text { anormal }\end{array}$ \\
\hline $0-5$ & 20 & $13(65 \%)$ \\
$5-10$ & 12 & $5(41 \%)$ \\
$10-15$ & 4 & $2(50 \%)$ \\
$15-20$ & 6 & $5(83 \%)$ \\
\hline
\end{tabular}

Cuadro 1 - Resultados da gamaencefalometria em relação ao tempo de doenca.

\begin{tabular}{c|c|c|c}
\hline \multirow{2}{*}{ Eletrencefalograma } & \multirow{2}{*}{ Nümero de casos } & \multicolumn{2}{|c}{ Gamaencefalometria } \\
\cline { 3 - 4 } & 6 & Normal & Anormal \\
\hline Normal & 6 & 4 & $2(33 \%)$ \\
Anormalidades difusas & 30 & 9 & $21(33 \%)$ \\
Anormalidades focais & & $21 \quad(70 \%)$ \\
\hline
\end{tabular}

Quxdro 2 - Resultados da gamaencefalometria em relação aos exames eletrencefalográficos.

\begin{tabular}{c|c|c|c|c|c}
\hline \multirow{2}{*}{ Quadro clinico } & \multirow{2}{*}{$\begin{array}{c}N^{\circ} \text { de } \\
\text { casos }\end{array}$} & \multicolumn{2}{|c|}{ Eletrencefalograma } & \multicolumn{2}{|c}{ Gamaencefalometria } \\
\cline { 3 - 6 } & Normal & Anormal & Normal & Anormal \\
\hline Quadro convulsivo & 36 & 5 & 31 & 16 & $20(55 \%)$ \\
Sem quadro convulsivo & 6 & 1 & 5 & 0 & $6(100 \%)$ \\
\hline
\end{tabular}

Quadro 3 - Resultados da gamaencefalometria em relação aos quadros clínico $\epsilon$ eletrencefalográfico. 
Em 12 casos a absorpção foi do tipo lesño e, em 13, do tipo edema. Relacionando êsses casos com os resultados do eletrencefalograma, obtivemos os dados condensados no quadro 4:

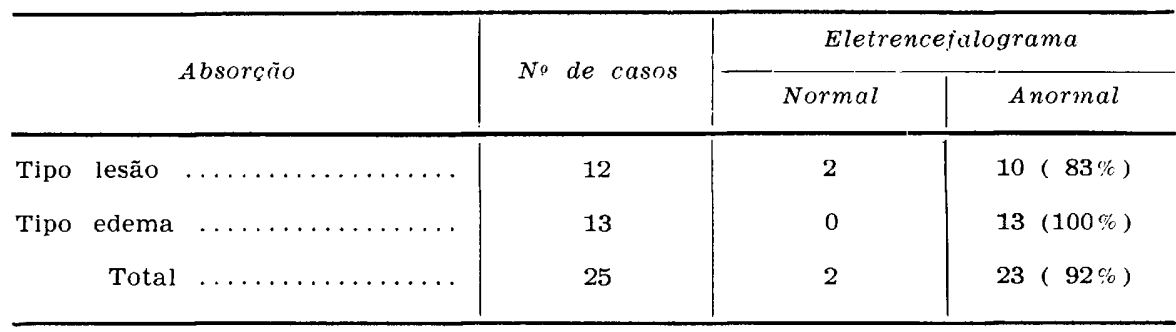

Quadro 4

Quanto à correspondência entre localização da lesão fornecida pelo EEG e pela GEM, em 14 casos houve concordância de hemisfério cerebral e em 9 a localizaçāo foi contralateral. Nos primeiros havia 4 casos com absorção tipo lesāo e 10 com absorção tipo edema; nos segundos, 6 tipo lesão e 3 tipo edema.

\section{COMENTARIOS}

Planiol e Fischgold, quando estudaram seus 126 casos, chamaram a atenção para o valor da GEM no diagnóstico das epilepsias condicionadas por tumores cerebrais, valorizando o exame apenas como segurança suplementar para afastar o diagnóstico de tais neoplasias. Admitindo como indiscutível o valor da GEM no diagnóstico dos tumores cerebrais, propusêmo-nos a estudar o método em 42 casos de epilepsia nos quais não havia sinais neurológicos focais nem hipertensão intracraniana.

A gamaencefalometria apresentou maior percentagem de anormalidades nos pacientes mais antigos, com evolução de mais de 15 anos (quadro 1); é improvável, levando-se em conta o longo tempo de evolução da moléstia, que se trate de processo tumoral. Nesses casos, outra teria de ser a explicação para as anormalidades encontradas na absorção do radioisótopo pelo tecido nervoso. Comparando os dados obtidos pela gamaencefalometria com aquêles fornecidos pela eletrencefalografia (quadro 2) verifica-se que a maior percentagem das anormalidades gamaencefalométricas se encontra entre os casos com alterações eletrencefalográficas focais; a menor percentagem ocorreu nos casos com anormalidades eletrencefalográficas difusas ou com EEG normal.

Por outro lado, é importante assinalar que em 33\% dos casos de EEG normal a absorção do radioisótopo foi anormal; partindo dêste dado, podemos admitir que a GEM poderá ser um recurso a mais para o diagnóstico de alguns casos de epilepsia, nos quais haja dúvida sob o ponto de vista clínico e nos quais o EEG seja normal. A comparação entre os dados clinico-eletrencefalográficos e os gamaencefalométricos (quadro 3) mostrou 
que as anormalidades gamaencefalomêtricas ocorreram em 100\% dos casos sem manifestações convulsivas e em apenas $55 \%$ dos casos que apresentavam convulsões. É geralmente admitido que, em casos graves de epilepsia convulsiva, a repetição das crises determina sofrimento cerebral que pode levar a alterações cerebrais estruturais; as crises com caracteristicas não convulsivas seriam menos capazes de provocar tais lesões ${ }^{1}$. Os resultados que obtivemos, admitindo-se que à maior absorção do radioisótopo corresponda uma área cerebral com grau maior ou menor de alteração, não confirmam essas idéias, pois a maior percentagem de anormalidades gamaencefalométricas ocorreu no grupo de epilepsias não convulsivas.

Dos 25 casos com GEM anormal, em 12 a absorção foi do tipo lesional e em 13 do tipo edematoso. Em 92\% dêsses casos o EEG foi anormal (quadro 4), sendo que a anormalidade eletrencefalográfica predominou nìtidamente no grupo em que a GEM sugeria existirem alterações de tipo edematoso $(100 \%)$. O resultado global não surpreende, pois é de esperar que $\mathrm{em}$ cérebros anormais ocorram anormalidades eletrencefalográficas; 0 que não tem explicação, pelo menos no estado atual de nossos conhecimentos, é a positividade maior dêsse exame nos casos em que houve absorção menos acentuada do radioisótopo.

A não correspondência das anormalidades eletrencefalográficas e gamaencefalométricas no que diz respeito à localização é explicável, em parte, pelo tempo decorrido entre um exame e outro que, em alguns casos, chegou a meses. Os casos em que houve coincidência de localização poderiam reforçar a idéia de um processo lesional mais grave; no entanto, dos 14 casos com anormalidades homolaterais, em 10 dêles a absorção era de tipo edematoso. Também para êste fato não encontramos, no momento, explicação.

Finalmente, deve ser assinalado, para registro, que no caso em que a pneumencefalografia foi anormal (atrofias corticais), a gamaencefalometria foi normal, ao passo que, no caso em que a pneumencefalografia foi normal, a gamaencefalometria foi anormal, tipo lesional.

Em conclusão: a maior absorção de radioisótopos revelada pela gamaencefalometria nem sempre indica, de maneira absoluta, a existência de processo expansivo; em nenhum dos 42 casos apresentados havia elementos clínicos indicadores da presença de tumor e, no entanto, a GEM foi positiva em alta percentagem neste grupo de pacientes. Esses resultados demonstram a importância da GEM nas epilepsias, na verificação de alterações estruturais encefálicas não tumorais. As anormalidades gamaencefalométricas nos casos com EEG normal sugerem a possibilidade do uso dêste exame no diagnóstico diferencial entre epilepsia e neurose. As anormalidades eletrencefalográficas predominaram nìtidamente no grupo com alterações gamaencefalométricas de tipo edematoso. Entretanto, para que se faça um juízo mais definitivo do valor dêsse método de investigação nas epilepsias é necessário, além de maior número de casos, um estudo paralelo sob o ponto de vista radiológico contrastado. De qualquer forma, sendo um exame in- 
cruento, de fácil realização em ambulatório, está indicado em todos os casos de epilepsia nos quais seja exigida melhor documentação para o diagnóstico definitivo.

\section{RESUMO}

Os autores estudam os resultados da gamaencefalometria em 42 epilépticos com mais de 10 anos de idade, sem sinais neurológicos focais ou hipertensão intracraniana. É ressaltada a importância dêsse exame nas epilepsias, na verificação de alterações estruturais não tumorais. A existência de $33 \%$ de anormalidades gamaencefalométricas nos casos de EEG normal sugere a possibilidade do emprêgo da gamaencefalometria no diagnóstico diferencial entre epilepsia e neurose. Entretanto, para que se possa tirar uma conclusão mais definitiva é necessário, além de um número maior de casos, estudo radiológico contrastado concomitante. De qualquer forma, pela sua fácil execução e inocuidade, esta a gamaencefalometria indicada em todos os casos de epilepsia que exijam melhor documentação.

\section{SUMMARY}

The importance of gamaencephalometry in the epilepsies.

The authors study the results of gammaencephalometry in 42 epilepctics over 10 years of age, without focal neurological signs or intracranial hypertension. The importance of this examination in epilepsy is emphasized, because it detects non-tumoral structural alterations. The existence of $33 \%$ of gammaencephalometric anormalities in cases with a normal EEG suggests the possibility of using gammaencephalometry in the differential diagnosis between epilepsy and neurosis. Nevertheless, in order to reach a more definite conclusion, it is necessary to study a larger number of cases, inclusively using roentgenography with a contrast medium. Anyway, owing to its hamlessness and feasibility, the authors think that gammaencephalometry is indicated in all cases of epilepsy that require full data.

\section{REFERENCIAS}

1. PENFIELD, W.; JASPER, H. - Epilepsy and the Functional Anatomy of the Human Brain. Little Brown Co., Boston, 1954, págs. 273-280. 2. PLANIOL, T.; FISCHGOLD, H. - Gama-encéphalographie et électroencéphalographie dans le diagnostic étiologique de l'epilepsie de l'adulte. In Fischgold, H.; Gastaut, H.: Rayons X. Radio-isotopes et EEG dans l'Epilepsie. Masson \& Cie., Paris, 1960, págs. 219-237. 